\title{
Relativistic Paschen-Back Effect for the Two-Dimensional H-Like Atoms
}

\author{
A. Poszwa* And A. Ruthowski ${ }^{\dagger}$ \\ Department of Physics and Computer Methods, University of Warmia and Mazury in Olsztyn \\ Żołnierska 14, 10-561 Olsztyn, Poland
}

(Received October 26, 2009; in final form January 15, 2010)

\begin{abstract}
The classification of states based on good quantum numbers for the two-dimensional Coulomb problem is proposed. The first order magnetic energy corrections are calculated using exact field-free analytic solutions of the Dirac equation as a zero-order approximation.
\end{abstract}

PACS numbers: 03.65.Pm, 03.65.Ge, 31.15.-p, 68.65.Fg

\section{Introduction}

Low-dimensional quantum systems have been the focus of extensive theoretical investigations in the last decades. Technological advances in semiconductor physics and recent developments in nanostructure technology provide techniques of creating low-dimensional structures like superlatices, quantum dots, quantum wires, or quantum wells [1]. The most representative analogues of hydrogen-like systems in the world of semiconductors are hydrogenic donors being the bound states of conduction electron and a donor impurity [2] and the Wannier-Mott excitons formed by an electron and a hole [3]. After the renormalization of Coulomb potential by introducing of dielectric constant and replacing the electron mass by effective one, the atomic objects in two-dimensional structures can be treated as 2D hydrogen-like atoms. At this stage, the 2D hydrogen problem determines a leading approximation for study of hydrogen type bound states in extreme anisotropic crystals, in which the $z$ component of a diagonal anisotropic mass tensor is much larger than the two remaining ones [4]. The non- and weak-relativistic [5] approaches are usually considered as sufficiently good approximations to the realistic description of the 2D objects in solid matter. However, in a searching of both spin and relativistic effects the complete relativistic theory based on the Dirac equation is inevitable. The first-order relativistic correction for wide range of magnetic fields have been calculated recently in the framework of direct perturbation theory (DPT) [6].

The aim of this paper is the calculation of the first-

\footnotetext{
* e-mail: poszwa@matman.uwm.edu.pl

$\dagger$ e-mail: andrut@matman.uwm.edu.pl
}

-order magnetic energy correction using fully relativistic wave functions as a zero-order approximation in the perturbation method.

\section{Exact solution for field-free atom}

The purpose of this section is the analysis of integrals of motion of the two-dimensional relativistic hydrogen atom and classification of the states based on good quantum numbers. These goals are achieved by introducing, into Dirac Hamiltonian, Hermitian operators associated with conserved quantities. The quantum mechanical two-dimensional central problem, with the Coulomb potential $-Z / \rho$ has been solved by many authors. In the nonrelativistic theory the analytic solution can be derived in strict analogy to the three-dimensional Coulomb problem, after the separation of the Schrödinger equation in polar coordinates $[7,8]$ or in parabolic coordinates [9]. The solution of the relativistic 2D hydrogen-like problem has been obtained in the framework of the two-component approach [10]. Although the formalism based on the two-dimensional representation of gamma matrices gives correct formula for energy levels, it does not provide a good background for analysis of other observables. Alternatively, standard Dirac-Pauli representation of the Dirac matrices can be used. The four-component analytic solution of the Dirac equation with the Hamiltonian

$$
H=c \boldsymbol{\alpha} \cdot \boldsymbol{p}+\beta c^{2}-\frac{Z}{\rho}
$$

in two spatial dimensions has been obtained by Guo et al. [11]. These authors have investigated two decoupled subspaces of the energy eigenstates

$$
\Psi^{(1)}=\left[\begin{array}{c}
f_{1}(\rho) \mathrm{e}^{\mathrm{i}(\mu-1 / 2) \phi} \\
0 \\
0 \\
\mathrm{i} g_{1}(\rho) \mathrm{e}^{\mathrm{i}(\mu+1 / 2) \phi}
\end{array}\right],
$$




$$
\Psi^{(2)}=\left[\begin{array}{c}
0 \\
f_{2}(\rho) \mathrm{e}^{\mathrm{i}(\mu+1 / 2) \phi} \\
\mathrm{i} g_{2}(\rho) \mathrm{e}^{\mathrm{i}(\mu-1 / 2) \phi} \\
0
\end{array}\right],
$$

where $\mu$ is the eigenvalue of the $z$-component of the total angular momentum $j_{z}$ defined as:

$$
j_{z}=l_{z}+\frac{1}{2} \sigma_{z}^{\prime}
$$

where

$$
l_{z}=-\mathrm{i} \partial / \partial \phi, \quad \sigma_{z}^{\prime}=\left[\begin{array}{cc}
\sigma_{z}^{\prime} & 0 \\
0 & \sigma_{z}
\end{array}\right] .
$$

In nonrelativistic limit the states of the form (2) describe an electron with spin up and down, respectively. Without any additional information about other conserved quantities the radial amplitudes of these two decoupled states have to be determined by two different sets of radial equations [11]. One of the important aspects of the presented approach is the possibility of determining all radial functions using one two-component radial equation.

We have find that two linearly-independent states (2) are the eigenstates of an operator

$$
P=\beta \sigma_{z}^{\prime},
$$

which commute with the Hamiltonian (1). It follows from (5) that $P$ is an involution $\left(P^{2}=1\right)$ and has two eigenvalues $\eta= \pm 1$. In the nonrelativistic limit the different signs of $\eta$ correspond to states with opposite spin directions. Moreover, it can be directly verified, by computing relevant commutators, that beyond operators $j_{z}$ and $P$ there exists an operator $K$ :

$$
K=\beta\left(\sigma_{z}^{\prime} l_{z}+\frac{1}{2}\right),
$$

which commutes with $H$ and both operators $P, j_{z}$. The eigenvalue of $K$ can be referred to as the Dirac quantum number $\kappa$. To determine a physical meaning of quantum number $\kappa$, let us derive a relation between $\kappa$ and $\mu$. If we consider the square of $K$ we obtain

$$
K^{2}=\left(\sigma_{z}^{\prime} l_{z}\right)^{2}+\sigma_{z}^{\prime} l_{z}+\frac{1}{4}=\left(l_{z}+\frac{1}{2} \sigma_{z}^{\prime}\right)^{2}=j_{z}^{2},
$$

which means that eigenvalues of $K$ satisfy the relation

$$
\kappa= \pm|\mu| \text {. }
$$

We note that operators $K, P$ and $j_{z}$ are not independent. They fulfil the relation

$$
K=P j_{z},
$$

which gives a similar relation for eigenvalues

$$
\kappa=\mu \eta \text {. }
$$

It appears from (10) that the upper and lower signs in (8) distinguish between two different physical situations, when, in nonrelativistic limit, spin is parallel or antiparallel to the total angular momentum.

Now we are in a position to solve the 2D Coulomb problem in a strict analogy to 3D case and perform the classification of states free of nonrelativistic quantum numbers. To this end we introduce to the Dirac equation the quantum numbers associated with the complete set of commuting operators $\left(H, K, j_{z}\right)$.

In atomic units and polar coordinates $(\rho, \phi)$ Hamiltonian (1) can be written in the form

$$
H=c\left(\alpha_{\rho} \pi_{\rho}+\frac{\mathrm{i}}{\rho} \alpha_{\rho} \beta K\right)+\beta c^{2}-\frac{Z}{\rho},
$$

where

$$
\begin{aligned}
& \alpha_{\rho}=\left[\begin{array}{cc}
0 & \boldsymbol{\sigma} \cdot \hat{\boldsymbol{\rho}} \\
\boldsymbol{\sigma} \cdot \hat{\boldsymbol{\rho}} & 0
\end{array}\right], \quad \boldsymbol{\sigma} \cdot \hat{\boldsymbol{\rho}}=\left[\begin{array}{cc}
0 & \mathrm{e}^{-\mathrm{i} \phi} \\
\mathrm{e}^{\mathrm{i} \phi} & 0
\end{array}\right], \\
& \pi_{\rho}=-\mathrm{i}\left(\frac{\partial}{\partial \rho}+\frac{1}{2 \rho}\right) .
\end{aligned}
$$

In the representation in which operators $H, j_{z}$ and $K$ are diagonal, energy levels are determined by radial part of the wave function only. The pertinent radial Dirac equation takes the form

$$
\left[c\left(\alpha_{\rho} \pi_{\rho}+\frac{\mathrm{i} \kappa}{\rho} \alpha_{\rho} \beta\right)+\beta c^{2}-\frac{Z}{\rho}\right] R=W R .
$$

Since $\alpha_{\rho}$ and $\beta$ fulfil the relations

$$
\alpha_{\rho}^{2}=\beta^{2}=1, \quad \alpha_{\rho} \beta+\beta \alpha_{\rho}=0,
$$

they can be represented by two-dimensional Hermitian matrices

$$
\alpha_{\rho}=\left[\begin{array}{cc}
0 & -\mathrm{i} \\
\mathrm{i} & 0
\end{array}\right], \quad \beta=\left[\begin{array}{cc}
1 & 0 \\
0 & -1
\end{array}\right] .
$$

According to Eq. (15) the radial function $R$ has two components, which for convenience can be taken in the form

$$
R(\rho)=\frac{1}{\rho^{1 / 2}}\left[\begin{array}{c}
F(\rho) \\
G(\rho) Z / c
\end{array}\right] .
$$

Introducing two new variables

$$
r=Z \rho, \quad E=\frac{W-c^{2}}{Z^{2}}
$$

and substituting (15) into (13) leads to the wave equation for the electron moving in the two-dimensional Coulomb field in the form of the pair of $\kappa$-dependent radial equations

$$
\begin{aligned}
& \frac{\mathrm{d} G}{\mathrm{~d} r}+\frac{\kappa}{r} G+\left(\frac{1}{r}+E\right) F=0, \\
& \frac{\mathrm{d} F}{\mathrm{~d} r}-\frac{\kappa}{r} F-\left[\lambda\left(\frac{1}{r}+E\right)+2\right] G=0,
\end{aligned}
$$

where $\lambda=(Z / c)^{2}$.

Since asymptotic solutions of Eqs. (18), (19) decay exponentially, we try to find the solution of radial Eqs. (18) and (19) in the form

$$
F=r^{\gamma} \mathrm{e}^{-\alpha r} \sum_{i=0}^{\infty} a_{i} r^{i}, \quad G=r^{\gamma} \mathrm{e}^{-\alpha r} \sum_{i=0}^{\infty} b_{i} r^{i},
$$

where

$$
\alpha=\sqrt{-E(2+\lambda E)} .
$$

Substituting expansions (20) into Eqs. (18), (19) we obtain the linear relations between the expansion coefficients 


$$
\begin{aligned}
& (i+\gamma-\kappa) a_{i}-\lambda b_{i}=\left(E a_{i-1}-\alpha b_{i-1}\right) \frac{\alpha}{E}, \\
& a_{i}+(i+\gamma+\kappa) b_{i}=-E a_{i-1}+\alpha b_{i-1} .
\end{aligned}
$$

For $i=0$, we have

$$
(\gamma-\kappa) a_{0}-\lambda b_{0}=0, \quad a_{0}+(\gamma+\kappa) b_{0}=0 .
$$

Since both $a_{0}$ and $b_{0}$ are different from zero, the secular determinant must vanish, which leads to

$$
\gamma= \pm \sqrt{\kappa^{2}-\lambda}
$$

and

$$
b_{0}=-\frac{a_{0}}{\gamma+\kappa} .
$$

For $i>0$, the expanding coefficients can be calculated iteratively from the relations (22) and (23). The condition of square integrability of the wave function allows only the upper sign in Eq. (25) and requires the termination of power series (20) at a some power $n^{\prime}$ :

$$
a_{n^{\prime}+1}=0, \quad b_{n^{\prime}+1}=0, \quad a_{n^{\prime}} \neq 0, \quad b_{n^{\prime}} \neq 0,
$$

which leads to the condition

$$
E a_{n^{\prime}}=\alpha b_{n^{\prime}},
$$

and finally to the equation for $E$ :

$$
\sqrt{-E(2+\lambda E)}\left(n^{\prime}+\gamma\right)=1+\lambda E .
$$

Solving this equation and substituting

$$
\gamma=\sqrt{\kappa^{2}-\lambda}
$$

we obtain

$$
E=\frac{1}{\lambda}\left[\left(1+\frac{\lambda}{\left(n^{\prime}+\sqrt{\kappa^{2}-\lambda}\right)^{2}}\right)^{-1 / 2}-1\right] .
$$

In order to compare the expression (31) with nonrelativistic one, we define the principal quantum number as follows:

$$
n=n^{\prime}+|\kappa|+1 / 2 .
$$

Since $n^{\prime} \geq 0$ we must have $|\kappa| \leq(n-1 / 2)$. However, for $n^{\prime}=0$, the number $\kappa$ must have positive value only. The absence of the $\kappa<0$ for $n^{\prime}=0$ follows from Eqs. (26) and (28), which both imply

$$
(\gamma+\kappa)>0 \text {. }
$$

According to (30), $\gamma$ is a real number smaller than $|\kappa|$ and inequality (33) can be satisfied only if $\kappa$ is positive. Therefore $\kappa$ must fulfil the relation

$$
\left|\kappa-\frac{1}{2}\right| \leq n-1 \text {. }
$$

Finally, energy levels in hartree are given through

$$
\begin{aligned}
& E_{n \kappa}=\left[\left(1+\frac{\lambda}{\left(n-|\kappa|-1 / 2+\sqrt{\kappa^{2}-\lambda}\right)^{2}}\right)^{-1 / 2}-1\right] \\
& \quad / \lambda .
\end{aligned}
$$

In the nonrelativistic limit $(\lambda \rightarrow 0)$ we obtain

$$
E_{n}=-\frac{2}{(2 n-1)^{2}} \text {. }
$$

The complete spin-space dependence of wave functions is given by

$$
\Psi_{n \kappa \mu}(r, \phi)=\frac{1}{r^{1 / 2}}\left[\begin{array}{c}
F_{n \kappa}(r) \Omega_{\kappa \mu}(\phi) \\
G_{n \kappa}(r) \Omega_{-\kappa \mu}(\phi)
\end{array}\right],
$$

where

$$
\begin{aligned}
& n=1,2,3, \ldots, \\
& \kappa=1 / 2,-1 / 2,3 / 2,-3 / 2, \ldots,(n-1 / 2),
\end{aligned}
$$

and

$$
\mu= \pm \kappa \text {. }
$$

The cylindrical spinor $\Omega_{\kappa \mu}(\phi)$ is defined as:

$$
\Omega_{\kappa \mu}(\phi)=\left[\begin{array}{c}
\frac{\kappa+\mu}{2 \mu} \mathrm{e}^{\mathrm{i}(\mu-1 / 2) \phi} \\
\frac{-\kappa+\mu}{2 \mu} \mathrm{e}^{\mathrm{i}(\mu+1 / 2) \phi}
\end{array}\right],
$$

and radial amplitudes have the form

$$
\begin{aligned}
& F_{n \kappa}(r)=r^{\gamma} \mathrm{e}^{-\alpha r} \sum_{i=0}^{n-|\kappa|-1 / 2} a_{i} r^{i}, \\
& G_{n \kappa}(r)=r^{\gamma} \mathrm{e}^{-\alpha r} \sum_{i=0}^{n-|\kappa|-1 / 2} b_{i} r^{i},
\end{aligned}
$$

with coefficients determined by relations (22) and (23).

Alternatively, in a similar way as in $3 \mathrm{D}$ case, the solution of radial Eqs. (18) and (19) may be expressed in terms of confluent hypergeometric functions

$$
\begin{aligned}
& F_{n \kappa}(r)=r^{\gamma} \mathrm{e}^{-\alpha r}\left[\left(\kappa+\frac{1}{\alpha}\right) F_{1}(r)-n^{\prime} F_{2}(r)\right], \\
& G_{n \kappa}(r)=\frac{E}{\alpha} r^{\gamma} \mathrm{e}^{-\alpha r}\left[\left(\kappa+\frac{1}{\alpha}\right) F_{1}(r)+n^{\prime} F_{2}(r)\right],
\end{aligned}
$$

where

$$
\begin{aligned}
& F_{1}(r)={ }_{1} F_{1}\left(-n^{\prime}, 2 \gamma+1 ; 2 \alpha r\right), \\
& F_{2}(r)={ }_{1} F_{1}\left(1-n^{\prime}, 2 \gamma+1 ; 2 \alpha r\right) .
\end{aligned}
$$

In order to introduce the classification scheme based on spectroscopic notation it is useful to define an orbital quantum number as

$$
l=\left|\kappa-\frac{1}{2}\right| .
$$

Let us note that this quantity according to (34) satisfies the inequality $l \leq(n-1)$.

In Table I we display, as an example, the lowest few states with principal quantum numbers $n=1,2,3$. We can see that for a given $n$, the states with the same $|\kappa|$ are degenerate and the lowest energy corresponds to minimal value of $|\kappa|$. According to (40), for each value of $\kappa$ there are two possible values of $\mu$. Therefore, the degree of degeneracy of energy levels is 2 for $\kappa=(n-1 / 2)$ and 4 for $|\kappa|<(n-1 / 2)$, respectively. It is worth to point out that, in a contrast to the three-dimensional case, due to the equality $|\kappa|=|\mu|$, the states with $|\mu|<|\kappa|$ do not occur. 
TABLE I

Relativistic quantum numbers, spectroscopic notation and energies in hartree for bound states with $n=1,2,3$ for the two-dimensional hydrogen atom. The values of energy are computed with $c=137.03599976$ [14].

\begin{tabular}{c|c|c|c|c|c}
\hline \hline$n$ & $n^{\prime}=n-|\kappa|-1 / 2$ & $\kappa$ & $l=\left|\kappa-\frac{1}{2}\right|$ & Notation & Energy \\
\hline 1 & 0 & $1 / 2$ & 0 & $1 s_{1 / 2}$ & -2.000106514052 \\
2 & 1 & $1 / 2$ & 0 & $2 s_{1 / 2}$ & -0.222234057055 \\
2 & 1 & $-1 / 2$ & 1 & $2 p_{1 / 2}$ & -0.222234057055 \\
2 & 0 & $3 / 2$ & 1 & $2 p_{3 / 2}$ & -0.222223537086 \\
3 & 2 & $1 / 2$ & 0 & $3 s_{1 / 2}$ & -0.080002897124 \\
3 & 2 & $-1 / 2$ & 1 & $3 p_{1 / 2}$ & -0.080002897124 \\
3 & 1 & $3 / 2$ & 1 & $3 p_{3 / 2}$ & -0.080000624824 \\
3 & 1 & $-3 / 2$ & 2 & $3 d_{3 / 2}$ & -0.080000624824 \\
3 & 0 & $5 / 2$ & 2 & $3 d_{5 / 2}$ & -0.080000170405
\end{tabular}

TABLE II

First-order magnetic corrections $E^{(1)}$ to the energies for states with principal quantum numbers $n=1,2,3$ of the two-dimensional relativistic hydrogenic atoms. The upper and lower signs in the front of $E^{(1)}$ are referred to the two values of $\mu= \pm|\kappa|$, respectively. In the last column nonrelativistic values of linear magnetic corrections are given.

\begin{tabular}{|c|c|c|c|c|c|c|c|}
\hline \multirow[t]{2}{*}{$n^{\prime}$} & \multirow[t]{2}{*}{$\kappa$} & \multirow[t]{2}{*}{ State } & \multicolumn{4}{|c|}{$E^{(1)}$} & \multirow[t]{2}{*}{$E_{N}^{(1)}$} \\
\hline & & & $Z=1$ & $Z=20$ & $Z=40$ & $Z=60$ & \\
\hline \multirow[t]{3}{*}{0} & $1 / 2$ & $1 s_{1 / 2}$ & \pm 0.49997337 & \pm 0.48911266 & \pm 0.45297650 & \pm 0.37072192 & \pm 0.5 \\
\hline & $3 / 2$ & $2 p_{3 / 2}$ & \pm 0.99999112 & \pm 0.99644147 & \pm 0.98566260 & \pm 0.96733798 & \pm 1 \\
\hline & $5 / 2$ & $3 d_{5 / 2}$ & \pm 1.49999467 & \pm 1.49786813 & \pm 1.49145055 & \pm 1.48068021 & \pm 1.5 \\
\hline \multirow[t]{4}{*}{1} & $1 / 2$ & $2 s_{1 / 2}$ & \pm 0.49999704 & \pm 0.49879035 & \pm 0.49478025 & \pm 0.48576638 & \pm 0.5 \\
\hline & $-1 / 2$ & $2 p_{1 / 2}$ & $\mp 0.00000296$ & $\mp 0.00120965$ & $\mp 0.00521975$ & $\mp 0.01423362$ & 0 \\
\hline & $3 / 2$ & $3 p_{3 / 2}$ & \pm 0.99999680 & \pm 0.99871795 & \pm 0.99482266 & \pm 0.98815866 & \pm 1 \\
\hline & $-3 / 2$ & $3 d_{3 / 2}$ & \pm 0.49999680 & \pm 0.49871795 & \pm 0.49482266 & \pm 0.48815866 & \pm 0.5 \\
\hline \multirow[t]{2}{*}{2} & $1 / 2$ & $3 s_{1 / 2}$ & \pm 0.49999894 & \pm 0.49956759 & \pm 0.49818020 & \pm 0.49536264 & \pm 0.5 \\
\hline & $-1 / 2$ & $3 p_{1 / 2}$ & $\mp 0.00000107$ & $\mp 0.00043241$ & $\mp 0.00181980$ & $\mp 0.00463736$ & 0 \\
\hline
\end{tabular}

\section{Linear Paschen-Back effect}

Let us consider now the Dirac Hamiltonian describing transversal motion of an electron around a fixed center of the Coulomb field with charge $Z$ and in a static uniform magnetic field. In atomic units the relativistic Hamiltonian can be written in the form

$$
H=c \boldsymbol{\alpha} \cdot(\boldsymbol{p}+\boldsymbol{A})+\beta c^{2}-\frac{Z}{\rho} .
$$

Taking into account the standard four-dimensional Dirac-Pauli representation of the Dirac matrices and the vector potential $\boldsymbol{A}=\boldsymbol{B} \times \boldsymbol{\rho} / 2$ for $\boldsymbol{B}=B \hat{z}$ perpendicular to the plane of transversal motion of the electron, we can write

$$
\boldsymbol{\alpha} \cdot(\boldsymbol{p}+\boldsymbol{A})=\alpha_{\rho} p_{\rho}+\mathrm{i} \alpha_{\rho} \sigma_{z}^{\prime}\left(\frac{l_{z}}{\rho}+\frac{B \rho}{2}\right) .
$$

Introducing into Hamiltonian both operators $P$ and $K$, defined in a previous section, we obtain

$$
H=c\left(\alpha_{\rho} \pi_{\rho}+\frac{\mathrm{i}}{\rho} \alpha_{\rho} \beta K+\frac{\mathrm{i}}{2} B \rho \alpha_{\rho} \beta P\right)+\beta c^{2}-\frac{Z}{\rho}
$$

and appropriate radial Dirac equation in the form

$$
\begin{aligned}
& {\left[c\left(\alpha_{\rho} \pi_{\rho}+\frac{\mathrm{i} \kappa}{\rho} \alpha_{\rho} \beta+\frac{\mathrm{i} \eta B}{2} \rho \alpha_{\rho} \beta\right)+\beta c^{2}-\frac{Z}{\rho}\right] \mathcal{R}} \\
& \quad=W \mathcal{R} .
\end{aligned}
$$

Substituting (15) into (51) and changing variables according to (16) and (17) and introducing a new variable $B:=B / Z^{2}$ leads to the wave equation, for the electron moving in the superposition of the two-dimensional Coulomb field and constant homogeneous magnetic field, in the form of the pair of $\kappa$ - and $\eta$-dependent radial equations

$$
\frac{\mathrm{d} \mathcal{G}}{\mathrm{d} r}+\left(\frac{\kappa}{r}+\frac{1}{2} \eta B r\right) \mathcal{G}+\left(\frac{1}{r}+E\right) \mathcal{F}=0,
$$




$$
\frac{\mathrm{d} \mathcal{F}}{\mathrm{d} r}-\left(\frac{\kappa}{r}+\frac{1}{2} \eta B r\right) \mathcal{F}-\left[\lambda\left(\frac{1}{r}+E\right)+2\right] \mathcal{G}=0 .
$$

The complete spin-space description of eigenfunctions is the same as for the field-free atom. The only difference is in dependence of radial functions on both quantum numbers $\kappa$ and $\mu(\eta=\kappa / \mu)$. In consequence, magnetic energy shift may depend on the symmetry of the states. We investigate this problem in the first-order perturbation approach. In order to apply the perturbation formalism we rewrite Eqs. (52) and (53) in $2 \times 2$ matrix form

$$
\left(h^{(0)}+B h^{(1)}-E S\right) \Phi=0,
$$

where

$$
\begin{aligned}
& h^{(0)}=\left[\begin{array}{cc}
-1 / 2 & -\mathrm{d} / \mathrm{d} r-\kappa / r \\
\mathrm{~d} / \mathrm{d} r-\kappa / r & -(2+\lambda / r)
\end{array}\right], \\
& h^{(1)}=\left[\begin{array}{cc}
0 & -\eta r / 2 \\
-\eta r / 2 & 0
\end{array}\right], \quad S=\left[\begin{array}{ll}
1 & 0 \\
0 & \lambda
\end{array}\right], \\
& \Phi(r)=\left[\begin{array}{l}
\mathcal{F}(r) \\
\mathcal{G}(r)
\end{array}\right] .
\end{aligned}
$$

Perturbation expansions for energy and wave function

$$
E=\sum_{i=0}^{\infty} E^{(i)} B^{i}, \quad \Phi=\sum_{i=0}^{\infty} \Phi^{(i)} B^{i}
$$

lead to the following perturbation equations:

$$
h^{(0)} \Phi^{(n)}+h^{(1)} \Phi^{(n-1)}-\sum_{i=0}^{n} E^{(i)} S \Phi^{(n-i)}=0 .
$$

The zero-order equation $(n=0)$

$$
\left(h^{(0)}-E^{(0)} S\right) \Phi^{(0)}=0
$$

is equivalent to the system of radial Eqs. (18) and (19) for field-free atom. Under the condition of orthogonality

$$
\left\langle\Phi^{(0)}, S \Phi^{(i)}\right\rangle=0
$$

for $i>0$, the $n$-th order energy correction can be written in the form

$$
E^{(n)}=\frac{\left\langle\Phi^{(0)}, h^{(1)} \Phi^{(n-1)}\right\rangle}{\left\langle\Phi^{(0)}, S \Phi^{(0)}\right\rangle} .
$$

The calculation of the first-order energy correction

$$
E^{(1)}=-\eta \frac{\langle F, r G\rangle}{\langle F, F\rangle+\lambda\langle G, G\rangle}
$$

can be performed in closed form using radial functions (44) and (45). For $n^{\prime}=0(\kappa=|\mu|)$, we obtain

$$
E^{(1)}=\frac{\mu}{4 \kappa}(2 \gamma+1) \text {. }
$$

Taking into account that hypergeometric functions (46) depend only on $|\kappa|$, the dependence of functions $F$ and $G$ on the sign of $\kappa$ is due to the factor $(\kappa+1 / 2)$ in $(44)$ and (45). It means that for $n^{\prime} \geq 0$ the first-order energy corrections have the general form

$$
E^{(1)}=-\eta \frac{a \kappa+b}{g \kappa+d}=\mu A_{1}+\frac{\kappa}{\mu} A_{2},
$$

where

$$
A_{1}=\frac{a d-b g}{\kappa^{2} g^{2}-d^{2}}, \quad A_{2}=\frac{b d-\kappa^{2} a g}{\kappa^{2} g^{2}-d^{2}},
$$

and we have taken into account that $\eta=\mu / \kappa=\kappa / \mu$. Radial integrals $a, b, g, d$ depend only on $\kappa^{2}$ and $n^{\prime}$ and are given in Appendix. It appears from Eqs. (63) and (64) that the $\kappa$ - and $\mu$-degeneracy of field-free levels is completely removed by external magnetic field.

Since in nonrelativistic limit $\lambda=0$ and $G=$ $\frac{1}{2}\left(\mathrm{~d} F / \mathrm{d} r-\frac{\kappa}{r} F\right)$ we have $\langle F, r G\rangle=-\frac{1}{2}\left(\kappa+\frac{1}{2}\right)\langle F, F\rangle$ and in consequence

$$
E_{N}^{(1)}=\frac{\eta}{2}\left(\kappa+\frac{1}{2}\right)=\frac{1}{2}\left(\mu+\frac{\mu}{2 \kappa}\right) .
$$

Expressing the relativistic quantum numbers $\mu$ and $\kappa$ by the nonrelativistic quantum numbers $m$ and $m_{s}$ (the eigenvalues of $l_{z}$ and $s_{z}=\frac{1}{2} \sigma_{z}$ ) we have $\mu=m+m_{s}$ and $\frac{\mu}{2 \kappa}=m_{s}$, and finally

$$
E_{N}^{(1)}=\left(m+2 m_{s}\right) / 2 .
$$

The nonrelativistic formula (67) may be also derived directly from the Schrödinger-Pauli equation. It has the same form as in the 3D case (see for example [12]) and is independent of $Z$. The independence of $Z$ of the correction (67) follows from the simple scaling relation $E(Z, B)=Z^{2} E\left(1, B / Z^{2}\right)$ fulfilled by the nonrelativistic energy [13].

Let us consider the $Z$-dependence of the relativistic correction to the Paschen-Back effect, defined as

$$
\delta E=E^{(1)}-E_{N}^{(1)} .
$$

Taking into account Eqs. (63) and (66), we can find the analytic formula for the correction (68) for states $1 s_{1 / 2}, 2 p_{3 / 2}, 3 d_{5 / 2}, \ldots$, corresponding to the lowest states with $\kappa>0\left(n^{\prime}=0\right)$ :

$$
\delta E=\frac{\mu}{2|\mu|}\left(\sqrt{\mu^{2}-(Z / c)^{2}}-|\mu|\right) .
$$

Since the minimal absolute value of $\mu$ is $1 / 2$, the condition $\mu^{2}-(Z / c)^{2}>0$ leads to the restriction for possible values of the atomic numbers $Z \lesssim Z_{0}$, where $Z_{0} \approx 137 / 2$. For ground state $(\mu=-1 / 2)$ and for $Z$ close to $Z_{0}$ the first term in (69) is negligible in comparison with $|\mu|$ and the relativistic correction takes the maximal value $\delta E \approx 0.25$, which gives a half of the nonrelativistic Paschen-Back shift. For excited $2 p_{3 / 2}$ and $3 d_{5 / 2}$ states the influence of the relativity on the behavior of the Paschen-Back effect is less pronounced than for the states $1 s_{1 / 2}$. Nevertheless, one can also observe the $Z$-dependence of the relativistic Paschen-Back shifts. For other states the relativistic corrections to the Paschen-Back effect can be calculated numerically as a difference between Eqs. (64) and (67). Table II lists the first-order energy shifts of levels corresponding to states with $n=1,2,3$, presented in Table I. The maximal absolute error of each value does not exceed \pm 1 in the last digit. We can see that linear energy corrections $E^{(1)}$ strongly depend on the charge of the Coulomb center $Z$ and sign of both quantum numbers $\kappa$ and $\mu$. Since $E_{\mathrm{N}}^{(1)}$ is independent of $Z$, the observed $Z$-dependence of $E^{(1)}$ can be regarded as a purely relativistic effect. 


\section{Concluding remarks}

We have presented in this paper exact analytic solution of 2D Coulomb problem by introducing good quantum numbers into wave equation, and applying standard power series expansion method. The problem of energy spectrum is solved exactly in close analogy to the 3D case. Moreover, the wave functions are classified according to the complete set of constants of motion without appeal to the nonrelativistic limit and determined by one $\kappa$ dependent system of radial equations. Utilizing obtained solutions, we have investigated the first-order magnetic properties of the two-dimensional hydrogenic atoms. The results obtained in this work indicate that the relativistic effects are important and sharply increase with growing value of atomic number $Z$. The nonrelativistic energy shifts caused by the first-order magnetic field interaction are constant in this case. We expect a significant change of magnetic properties due to relativity also in higher orders of magnetic field interaction. The influence of relativity on the behavior of 2D hydrogenic atoms in magnetic field of arbitrary strength will be investigated in the forthcoming paper.

\section{Appendix}

Radial integrals appearing in the expression of the first-order magnetic energy correction (64) are defined as follows:

$$
\begin{aligned}
a & =\frac{2 E}{\alpha^{2}} K_{1}, \quad b=\frac{E}{\alpha}\left[\left(\kappa^{2}+\frac{1}{\alpha^{2}}\right) K_{1}-n^{\prime 2} K_{2}\right], \\
g & =\frac{2}{\alpha}\left(1+\frac{\lambda E^{2}}{\alpha^{2}}\right) I_{1}+2 n^{\prime}\left(\frac{\lambda E^{2}}{\alpha^{2}}-1\right) I_{12}, \\
d & =\left(1+\frac{\lambda E^{2}}{\alpha^{2}}\right)\left[\left(\kappa^{2}+\frac{1}{\alpha^{2}}\right) I_{1}+n^{\prime 2} I_{2}\right] \\
& +\frac{2 n^{\prime}}{\alpha}\left(\frac{\lambda E^{2}}{\alpha^{2}}-1\right) I_{12},
\end{aligned}
$$

$$
\begin{aligned}
& K_{i}=\int_{0}^{\infty} r^{2 \gamma+1} \mathrm{e}^{-2 \alpha r} F_{i}^{2} \mathrm{~d} r, \\
& I_{i}=\int_{0}^{\infty} r^{2 \gamma} \mathrm{e}^{-2 \alpha r} F_{i}^{2} \mathrm{~d} r,
\end{aligned}
$$

where $i=1,2$ and

$$
I_{12}=\int_{0}^{\infty} r^{2 \gamma} \mathrm{e}^{-2 \alpha r} F_{1} F_{2} \mathrm{~d} r .
$$

\section{References}

[1] A.D. Yoffe, Adv. Phys. 50, 1 (2001).

[2] Jia-Lin Zhu, Jia-Jiong Xiong, Bing-Lin Gu, Phys. Rev. B 41, 6001 (1990).

[3] J.A. Reyes, M. del Castillo-Mussot, Phys. Rev. B 57, 1690 (1998).

[4] X.G. Wen, A. Zee, Phys. Rev. Lett. 62, 1937 (1989); H.N. Spector, J. Lee, Am. J. Phys. 53, 248 (1985).

[5] V.M. Villalba, R. Pino, Physica E 10, 561 (2000).

[6] A. Rutkowski, A. Poszwa, Phys. Scr. 79, 065010 (2009).

[7] X.L. Yang, S.H. Guo, F.T. Chan, K.W. Wong, W.Y. Ching, Phys. Rev. A 43, 1186 (1991).

[8] B. Zaslow, M.E. Zandler, Am. J. Phys. 35, 1118 (1967).

[9] A. Cisneros, H.V. McIntosh, J. Math. Phys. 10, 277 (1969).

[10] Shi-Hai Dong, Zhong-Qi Ma, Phys. Lett. A 312, 78 (2003).

[11] S.H. Guo, X.L. Yang, F.T. Chan, K.W. Wong, W.Y. Ching, Phys. Rev. A 43, 1197 (1991).

[12] L.D. Landau, E.M. Lifshits, Quantum Mechanics, Pergamon, Oxford 1997.

[13] C.L. Surmelian, R.F. O'Connel, Astrophys. J. 190, 741 (1974).

[14] P.J. Mohr, B.N. Taylor, Rev. Mod. Phys. 72, 351 (2000).

where 